\title{
Lightness, brightness, and brightness contrast: 1. Illuminance variation
}

\author{
LAWRENCE E. AREND \\ Eye Research Institute, Boston, Massachusetts \\ and \\ BRANKA SPEHAR \\ Rutgers University, Newark, New Jersey
}

\begin{abstract}
Changes of annulus luminance in traditional disk-and-annulus patterns are perceptually ambiguous; they could be either reflectance or illuminance changes. In more complicated patterns, apparent reflectances are less ambiguous, letting us place test and standard patches on surrounds perceived to be different grays. Our subjects matched the apparent amounts of light coming from the patches (brightnesses), their apparent reflectances (lightnesses), or the brightness differences between the patches and their surrounds (brightness contrasts). The three criteria produced quantitatively different results. Brightness contrasts matched when the patch/surround luminance ratio of the test was approximately equal to that of the standard. Lightness matches were illumination invariant but were not exact reflectance matches; the different surrounds of test and standard produced a small illumination-invariant error. This constant error was negligible for increments, but, for decrements, it was approximately 1.5 Munsell value steps. Brightness matches covaried substantially with illuminance.
\end{abstract}

The research reported in this paper bears on several aspects of achromatic color perception.

1. At the most concrete level, we describe a new experimental paradigm in which observers quantitatively matched three distinct perceptual attributes of the same pattern-namely, brightnesses (apparent luminances), local brightness contrasts (brightness differences), and lightnesses (apparent reflectances).

2. More generally, we argue both from our data and on logical grounds that, over the past century, most experimenters who have tried to study perception of neutral surface color have used a fatally flawed paradigm. Their disk-and-annulus stimulus patterns were too simple and their subjects' tasks too vaguely defined to allow measurement of perceived surface colors.

3. At the most important and general level, we argue that sensory processes such as simultaneous contrast and adaptation are too simple to provide color-constant perception of surfaces. Early sensory processing must be followed by a more elaborate surface analysis (Adelson \& Pentland, 1991; Arend, 1990a, 1991, in press-b; Barrow \& Tennenbaum, 1978; Kersten, 1991; Marr, 1978). Sen-

We thank Alan L. Gilchrist for making B.S.'s visit possible and for valuable discussion. Paul Whittle and three anonymous reviewers provided numerous helpful suggestions. This research was supported by Air Force Office of Scientific Research Grants AFOSR 86-0128 and AFOSR 89-0377 to L.E.A. B.S. was supported by NSF Grant BNS8909182 to A. L. Gilchrist. Correspondence should be addressed to L. E. Arend, David Sarnoff Research Center, CN 5300, Princeton, NJ 08543-5300. sory processes probably play an important role in conditioning the visual system's representation of local luminance contrasts, but reliable perception of surface colors requires vector analysis of spatial gradients according to physical causes and integration of local luminance contrast information over multiple edges and gradients. Known sensory mechanisms provide neither.

We will use the term brightness to mean "apparent luminance" and the term lightness to mean "apparent reflectance."

\section{Prior Disk-and-Annulus Research}

Use of disk-and-annulus patterns in studies of achromatic vision dates back at least to Hess and Pretori's $(1894 / 1970)$ famous brightness contrast experiment. Over the past century, similar patterns have been used many times, but the results and interpretations continue to generate controversy (Gilchrist \& Jacobsen, 1989; Heinemann, 1989; Jacobsen \& Gilchrist, 1988). The analysis and data presented in the present series of papers are designed to clarify some of the issues behind the controversy. A new experimental paradigm eliminates some of the perceptual ambiguity of the disk-and-annulus patterns.

In his experiments on neutral surface colors, Hans Wallach used disk-and-annulus patterns and concluded that the apparent neutral surface color of the disk is primarily determined by the ratio of luminances at its immediate boundary (Wallach, 1948, 1976). He argued that the gray quality of a region is produced by local spatial interactions among neighboring luminances. He also thought that perceptions of neutral surface color are based on primary per- 
ceptual processes, but that perceptions of levels of illumination (shading, shadows, highlights) are not. Perceptions of illumination instead result from a quality that he called luminosity, which occurs when there is insufficient interaction at edges, with the type of illumination percept being determined by past experience (Wallach, 1976, p. 33).

Several more recent authors have extended this emphasis on local luminance relations as the primary determinant of neutral surface color (Evans, 1974; Heinemann, 1989; Shapley \& Enroth-Cugell, 1984).

In the present series of papers, we present an alternative view of the role of local luminance contrast in the perception of neutral colors. When added to previous work (e.g., Arend \& Goldstein, 1990; Gilchrist, Delman, \& Jacobsen, 1983), the data presented here provide strong evidence against local determination of apparent surface colors. Local luminance contrast determines local brightness contrast, not surface lightness, even in the simplest laboratory patterns.

In this first paper, we introduce our new patterns and tasks. When patch-and-surround patterns are embedded in a more complex display, perception of the patches includes three quantitatively and qualitatively distinct properties or dimensions. Observers can match brightnesses (apparent luminances), lightnesses (apparent reflectances), and local brightness contrasts (differences of brightness). When the test patches were on surrounds with different reflectances, the observers' luminance settings to match the three dimensions were three substantially different functions of illumination level. To avoid confusion, we asked the observer to do only one type of match within an experimental session, but all three properties were simultaneously visible.

In the second paper (Arend \& Spehar, 1993), we compare experiments with patch-and-surround patterns embedded within a complex pattern to experiments with isolated patch-and-surround patterns. The results clear up some confusion regarding earlier research with disk-andannulus patterns. Observers instructed to make lightness matches in isolated disk-and-annulus patterns match local brightness contrasts instead.

\section{Lightness and Brightness in Mondrian Patterns}

The experiments reported here are an extension of previous experiments on lightness and brightness in simple and complex achromatic patterns (Arend \& Goldstein, 1987b, 1990). In those experiments, the observers matched test patches in a pattern under one illuminant to standard patches in a pattern under a second illuminant. The lightness (apparent reflectance) matches were almost perfectly independent of illuminance; they demonstrated excellent lightness constancy.

The design of Arend and Goldstein's (1987b, 1990) experiments did not allow study of the role of local luminance contrast in lightness perception. In those experiments, the reflectance of the surround of the test patch was always equal to the reflectance of the surround of the standard patch. This was done to equate the reflec- tance environments of the patches, a desirable control As a consequence, however, luminance contrast and reflectance were correlated. Setting the reflectance of the test patch equal to that of the standard patch was physically identical to setting the luminance ratio at the edge of the test patch equal to that at the edge of the standard patch. Our instructions in the lightness-matching condition asked the subjects to "make the test and standard patches look like they were cut from the same piece of paper." Nevertheless, the physical linkage of reflectance and luminance ratio raises the possibility that our observers could have produced lightness-constant results by matching local brightness contrasts rather than apparent surface colors. One should also note that this alternative explanation requires the additional assumption that local brightness contrasts are equal when the local luminance contrasts are equal. This assumption, not always true, will be discussed further in connection with the data from our first experiment.

\section{New Stimulus Pattern}

Effective theoretical arguments can be made against the thesis that the lightness of a patch is determined primarily by the luminance contrast at its boundary, but we now also have data that clearly contradict that proposal. We have produced patterns in which the test and standard patches are surrounded by different reflectances. In the earlier experiments, we were able to unambiguously define two different tasks for our subjects, brightness matching and lightness matching. With these new stimuli, we were able to define a third task, brightness-contrast matching.

We used Mondrian patterns that had at the center two patches forming a patch $\left(\boldsymbol{P}_{\mathrm{tst}}, \boldsymbol{P}_{\mathrm{std}}\right)$ and surround $\left(\boldsymbol{S}_{\mathrm{tst}}\right.$, $S_{\text {std }}$ ) (Figure 1). For our present concerns, the most important virtue of this type of pattern is that it lets us do disk-and-annulus experiments within the context of a perceptually well-defined gray scale. That is, scattered about in the outer Mondrian region are patches that evenly populate a 33:1 luminance range. These appear to span the gray scale from black to white under uniform illumination. To the patch and surround, we assigned luminances within the range of the surrounding Mondrian. As a result, they had well-defined gray values in the sense that their relations to black and white were clear. Their apparent reflectances were less ambiguous than in the traditional diskand-annulus patterns, which do not, in general, span the gray scale.

This perceptually well-defined gray scale provided the means for separating lightness matching from brightnesscontrast matching. The lightness-matching task was the same as that in our original experiments. The subject produced a lightness match by adjusting the luminance of the test patch to "look like it was cut from the same paper" as the standard patch-that is, to give it the same apparent position in the gray scale as that of the standard patch. However, unlike in the original experiments, the test and standard patches were surrounded by different grays. As a result, at the equal reflectance setting, the $P_{\text {tst }} / S_{\text {tst }}$ lu- 


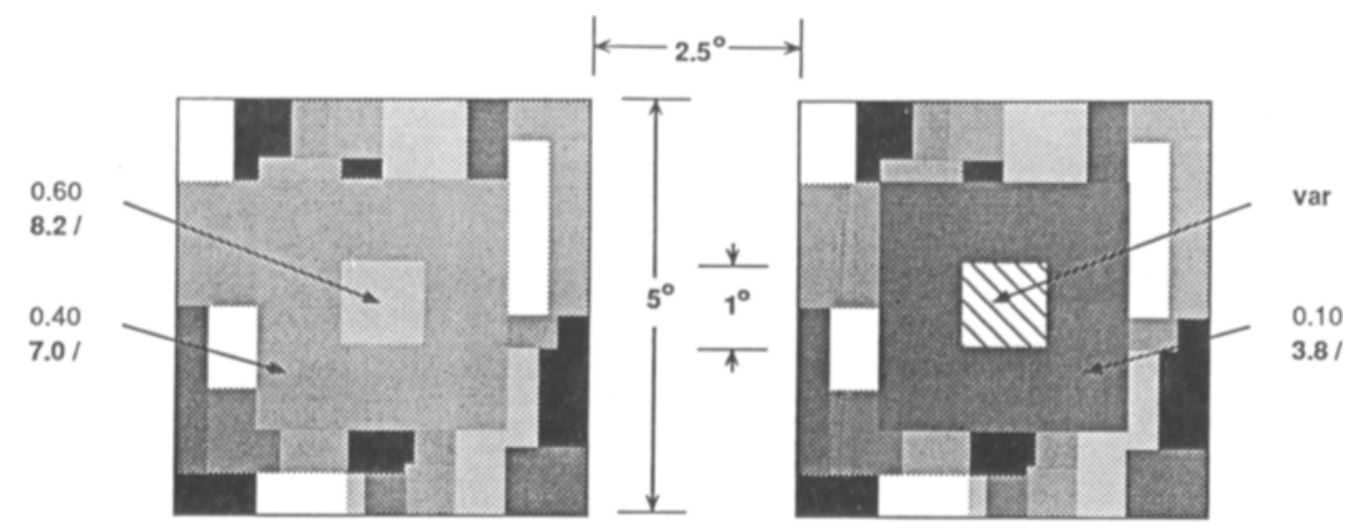

a.

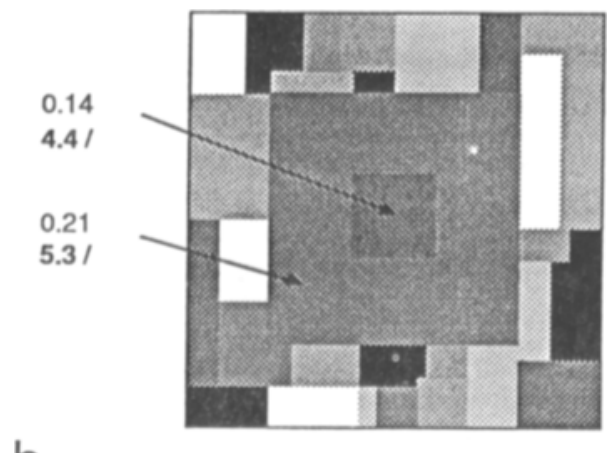

STANDARD

E Fixed

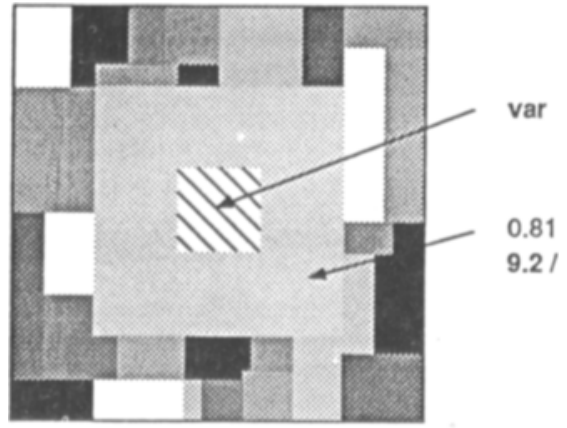

TEST

E Varied, $19: 1$

Figure 1. Diagrams of stimulus patterns. All patches were uniform matte regions on a color monitor. Plain type: reflectance. Boldface: Equivalent Munsell value. Reflectances were identical in Mondrian portions of test (right) and standard (left) arrays, ranging from 0.03 to 0.95 . The illuminance on the standard array was fixed. The illuminance on the test array varied from trial to trial, in five steps over a 19:1 range. The subjects varied the luminance of the test patch to match the standard patch by one of three criteria. (a) increment condition; (b) decrement condition.

minance ratio was not the same as the $P_{\text {std }} / S_{\text {std }}$ luminance ratio. Furthermore, at the subjects' equal lightness setting, the $P_{\text {tst }} / S_{\text {tst }}$ brightness difference was not the same as the $P_{\text {std }} / S_{\text {std }}$ brightness difference. When subjects were instructed to make the $P_{\text {tst }} / S_{\text {tst }}$ brightness difference equal to the $P_{\text {std }} / S_{\text {std }}$ brightness difference, they set the test patch to luminances substantially different from their lightness match luminances. Finally, the third task was the same brightness task that we had employed in our previous experiments. The subject was instructed to make the apparent amount of light coming from the test patch match that from the standard patch.

\section{Effects of Instructions}

The distinction between the lightness and brightness tasks has been referred to as an effect of instructions (Jameson \& Hurvich, 1989). In one sense this is true, of course, but it is important to distinguish among different types of effects of instructions. In the present context, the important distinction is that between instructions that alter the observers' perception of the display and those that merely indicate which of several simultaneously available aspects of a complicated percept is to be described. A particularly clear example of the former is the famous ambiguous girl-and-crone figure. Depending on expectations established by instructions, the observer is likely to see one of two percepts. The percepts are modal: while one is seen, the other is completely absent. On the other hand, the latter type of instruction effect involves no incompatibility of percepts and no substantial changes of percepts. A subject viewing the image of a three-dimensional faceted object can be instructed to report the apparent orientation of one of the faces or its apparent surface color. Although it is possible that the subject's attending to one 
dimension or another may slightly alter the appearance of the less attended dimension, these have typically been found to be second-order effects. The subject's overall percept of the image is affected little by the instruction.

Our lightness, brightness, brightness-contrast instructions are primarily of this second type. They simply tell the observer which dimension of the target to describe.

\section{Local Simultaneous Contrast Effects}

Before turning to the specific stimuli and data, we must consider one more consequence of the different surround reflectances in test and standard stimuli. The different surround reflectances may have differential local simultaneous contrast influences on the test and standard patches (Koffka, 1935; Takasaki, 1966; Whittle, in press-a, in press-b; Whittle \& Challands, 1969). It is well known that both the lightness and the brightness of a gray patch are affected by the reflectance of the immediately surrounding area. The common textbook figure that is used to demonstrate simultaneous brightness contrast consists of two medium gray patches in the centers of adjacent unprinted white and deepest black regions. The gray patch on the white side appears both darker gray and dimmer (less bright) than that on the black. Although the difference is small in comparison with those that occur in experiments with a wide range of inducing field luminances (e.g., Heinemann, 1955), Takasaki's experiment indicated that the effects can approach a difference of several Munsell value steps. Related effects in our data are analyzed in the Discussion section.

\section{METHOD}

\section{Equipment}

The gray patterns were presented on a carefully calibrated Tektronix 690SR high-resolution color monitor under the control of an Adage 3000 image processor and a VAX-11/750 minicomputer. Details of the apparatus and calibrations are given in a previous paper (Arend \& Reeves, 1986).

The gray-scale patterns had the same chromaticity $(6,500 \mathrm{~K})$ over the required luminance range and varied only in luminance, as programmed. There was no substantial chromaticity or luminance nonuniformity over the effective viewing area, and the display was stable within and between sessions. The functions relating digital data to luminance in the red, green, and blue channels of the imageprocessor/display system were measured. A linear relationship was obtained in each color channel through 10-bit look-up tables derived from the direct luminance measurements. To evaluate the resulting luminance curve output, luminances were directly measured. The curve was linear and accurate to very low luminances in all three guns.

The subjects controlled the luminance of the test patch by moving a hand-held cursor horizontally over a high-resolution graphics tablet. Between trials, the computer randomly offset the relationship between hand position and luminance within a range of $\pm 10 \%$, to prevent position cues from influencing the matches. The spatial resolution of the tablet exceeded the 10-bit resolution of the image processor's D/A converters.

\section{Stimuli}

The stimuli were simulations of uniformly illuminated matte papers lying in a common depth plane. For brevity, we will here- after drop the simulation terminology and refer to the stimuli and their simulated properties as though they were actual papers.

The stimuli are illustrated in Figure 1 . Two $1^{\circ}$-square patches were presented $7.5^{\circ}$ apart (center to center). Each patch was surrounded by a gray square surround whose inner border coincided with the edge of the patch and whose outer border subtended $3^{\circ}$. These uniform surrounds were each surrounded by a patchwork of small rectangles (a Mondrian), arranged in the shape of a square surround with a $3^{\circ}$-square inner border and a $5^{\circ}$-square outer border. The two patchwork arrays had identical spatial arrangements of 27 irregular rectilinear patches, with reflectances ranging from 0.03 to 0.95 (1.50 log units).

There were four reflectance conditions, with different combinations of patch and surround reflectances. Two of the conditions we will refer to as the control incremental and decremental conditions. In those conditions, the surrounds in the test and standard patterns had the same reflectance. They were therefore conceptual replications of our earlier experiments (Arend \& Goldstein, 1987b), differing only in details of the geometry of the patches.

In the experimental incremental and decremental conditions, the reflectance of the surround of the test array was different from that of the standard surround. We chose the reflectance combinations to give large separations of theoretical predictions for our three matching tasks (described below). In all four conditions, the subject adjusted the simulated reflectance of the test patch.

In the increment condition (Figure 1a), the standard patch and surround were light grays ( $R=0.60$; Munsell value $V=8.2 /$ and $R=0.40 ; V=7.0$, respectively). The test surround was a dark gray $(R=0.10 ; V=3.8 /)$ in the experimental condition and the same light gray $(R=0.40 ; V=7.0 /)$ as that of the standard surround in the control condition. In the decrement condition (Figure $1 \mathrm{~b})$, the standard patch and surround were dark grays $(R=$ $0.14 ; V=4.4 /$ and $R=0.21 ; V=5.3 /$, respectively). The test surround was white $(R=0.81 ; V=9.2 /)$ in the experimental condition and dark gray $(R=0.21 ; V=5.3)$ in the control condition. In the standard array, the ratio of patch-reflectance/surroundreflectance in the increment condition was the inverse of that in the decrement condition.

In all four reflectance conditions, reflectances of all patches but the test patch and the illuminance of the left pattern (standard array) were fixed within each session. The illuminance of the right pattern (test array) changed from trial to trial, in randomized blocks of five illuminances, spanning a range of $1.28 \mathrm{log}$ units in equal logarithmic steps. A 0.50 reflectance patch in the left pattern (standard) had a luminance of $23.5 \mathrm{~cd} / \mathrm{m}^{2}$ on all trials. The luminance, $L(\mathrm{p})$, of any particular patch, $\mathrm{p}$, in $\mathrm{cd} / \mathrm{m}^{2}$, is given by

$$
L(\mathrm{p})=\left[\frac{E_{\mathrm{tst}}}{E_{\mathrm{std}}}\right]\left(\frac{23.5}{0.50}\right) R(\mathrm{p})
$$

where $E_{\mathrm{tst}} / E_{\mathrm{std}}$ is the ratio of the illuminance on the test pattern (including $\mathrm{p}$ ) to that on the standard pattern, and $R(\mathrm{p})$ is the reflectance of $p$. The patterns appeared on a $14.2^{\circ}$-square, dark surround $\left(0.014 \mathrm{~cd} / \mathrm{m}^{2}, 0.006\right.$ of the luminance of the darkest patch of the standard Mondrian) in an otherwise completely darkened room.

\section{Procedure}

We are specifically interested in natural perception of relative surface color in scenes with spatially nonuniform illumination. We therefore rejected haploscopic viewing and successive viewing of test and standard patterns in favor of simultaneous binocular viewing. Some of the consequences of this choice are analyzed further in the Discussion section.

The subjects initially adapted to a $14.2^{\circ} \times 14.2^{\circ}$ visual angle, $6,500 \mathrm{~K}, 23.5-\mathrm{cd} / \mathrm{m}^{2}$ uniform white field for $3 \mathrm{~min}$. They then viewed the two continuously presented displays and matched the test patch in the right display with the corresponding standard patch in the left display, using the tablet to vary the test patch luminance. 
The match was made to satisfy one of three task criteria, described below. The subjects were asked to spend about the same amounts of time in looking at the test and standard patterns and to alternate their gaze between the patterns, shifting approximately once per $2 \mathrm{sec}$.

Five adjustments of one patch at each of the five illuminances, for one of the three tasks in both the incremental and the decremental conditions, constituted an experimental session, requiring approximately $20 \mathrm{~min}$. No more than two sessions per day per subject were run, separated by at least $15 \mathrm{~min}$ of rest time.

\section{Tasks}

There were three tasks (brightness, lightness, brightness contrast) in each of the experimental and control conditions.

In the lightness-matching condition, we instructed the subjects to make the test patch "look as if it were cut from the same piece of paper" as was the corresponding patch in the standard. It was pointed out that the outer, "Mondrian" region spanned a range of grays from black to white.

In the brightness-matching condition, we instructed the subjects to make the test patch "have the same brightness as the corresponding patch in the standard, disregarding, as much as possible, other areas of the display. That is, make the amount of light coming from the test patch look the same as that from the standard."

In the brightness-contrast task, we instructed the subjects to "make the brightness difference between the test patch and surround the same as that between the standard patch and surround."

\section{Subjects}

Three observers participated-the authors (L.A. and B.S.) and a paid observer (D.A.). B.S. was familiar with the purpose of the experiment but had no prior experience in lightness or brightness matching. D.A. was experienced in lightness and brightness matching but naive with respect to the purpose of the experiment.

\section{RESULTS}

The results for the control conditions (equal test and standard surround reflectances) are shown in Figure 2. The illumination level on the test array is indicated on the horizontal axis by the log of the ratio of the illuminance of the test array $\left(E_{\mathrm{tst}}\right)$ to the fixed illuminance $\left(E_{\mathrm{std}}\right)$ of the standard array. Thus the illuminations are equal at 0.0 , and $E_{1 \mathrm{st}}$ decreases to the left.

The subject adjusted the luminance of the test patch to make it match the standard patch, but it is convenient, for theoretical reasons, to plot the data as though the subject had adjusted the reflectance of the test patch. The subjects' mean log reflectance settings (mean log luminance minus log illuminance) are plotted as ordinates. For comparison, the Munsell values corresponding to the log reflectances of the left vertical axis are indicated on the right vertical axis. ${ }^{1}$

Results for the experimental conditions are shown in Figure 3. The data for increments (standard patch more luminous than the standard surround) are shown in the left panels; the data for decrements are shown in the right panels.

\section{Lightness Matches}

The circles in each panel in Figures 2 and 3 are the means for the lightness task. In the control condition (Figure 2), the two horizontal solid lines are theoretical, rep-

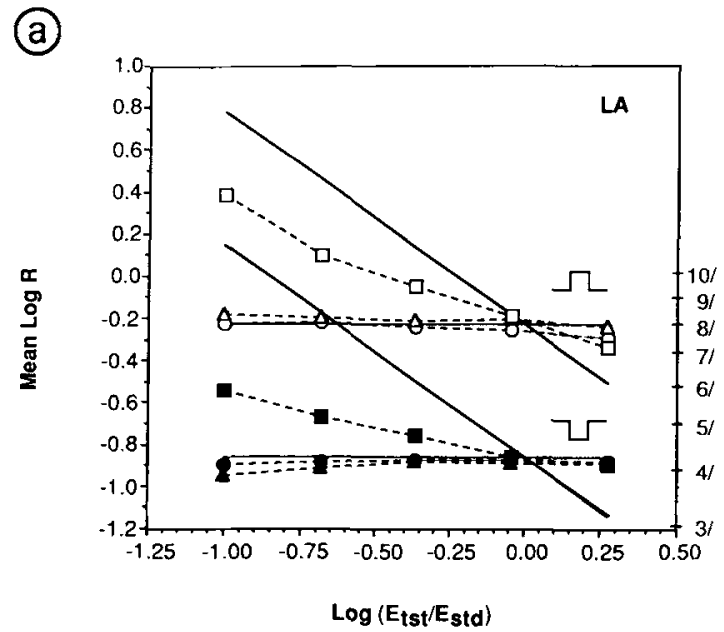

$\frac{9}{3}$
$\frac{10}{\overline{5}}$
$\overline{\bar{g}}$
$\frac{5}{3}$

(b)

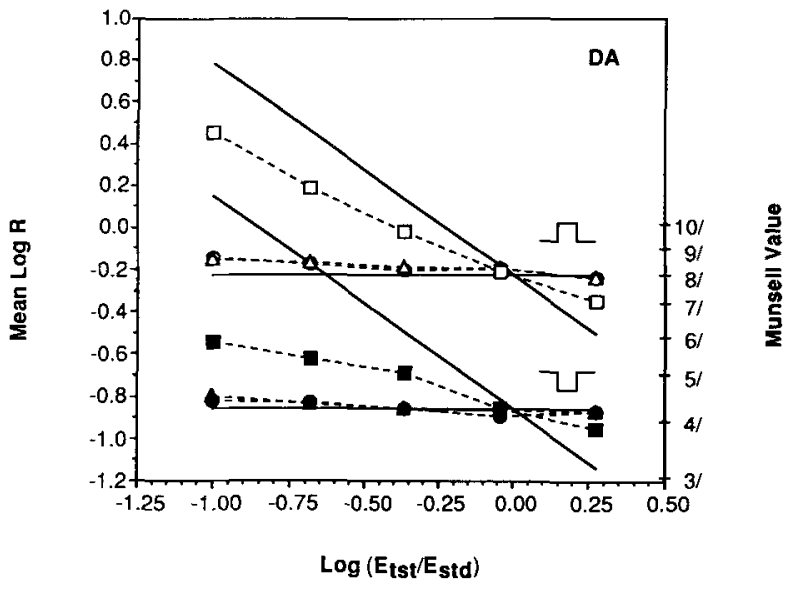

Figure 2. Mean log reflectance data for equal surround reflectances (control condition). Solid lines: theoretical lines, explained in text. Circles: lightness matches. Squares: brightness matches. Triangles: brightness-contrast matches. For all points, error bars $( \pm 1 S E)$ were smaller than the plot symbols. (a) Observer L.A. (b) Observer D.A.

resenting in each case perfect reflectance matches between the test and standard patches-that is, the perfect lightnessconstancy line. As in Arend and Goldstein's (1987b, 1990) earlier experiments with equal-reflectance surrounds, the subjects showed nearly perfect lightness constancy.

The top horizontal solid line in the increment condition (Figure 3, left panels) and the bottom horizontal solid line in the decrement condition (Figure 3, right panels) are the theoretical perfect lightness-constancy lines. In the increment condition, the data lie approximately on the theoretical line. In the decrement case, they lie approximately $0.2 \log$ units above the line, presumably because of local simultaneous contrast with the immediate surround. In both cases, the data lie along a nearly horizontal line, indicating approximate illumination invariance.

The greater local simultaneous contrast effect in the decrement condition is consistent with previous simultaneous 
(a)

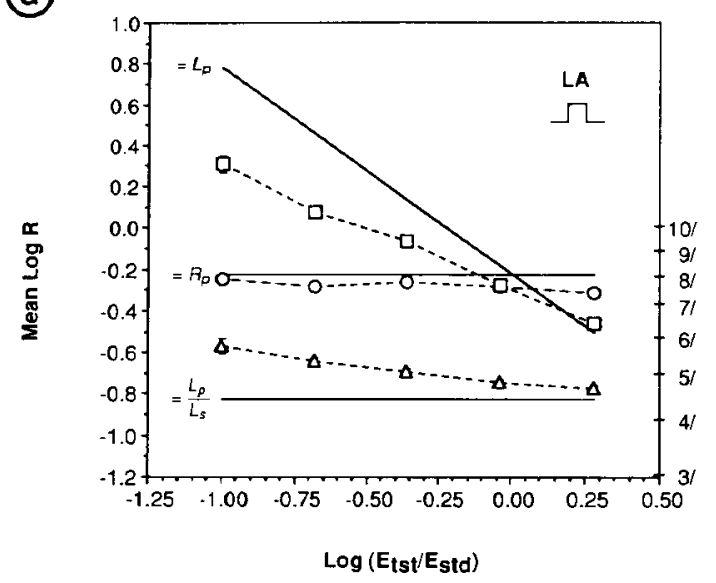

(b)

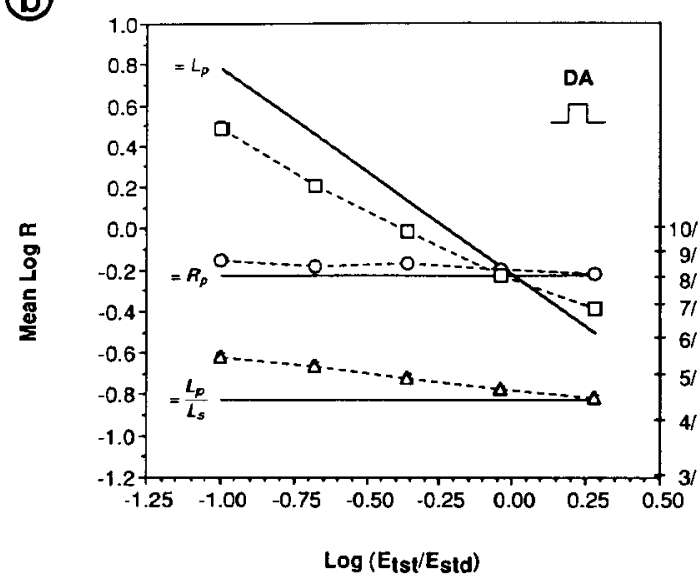

(C)

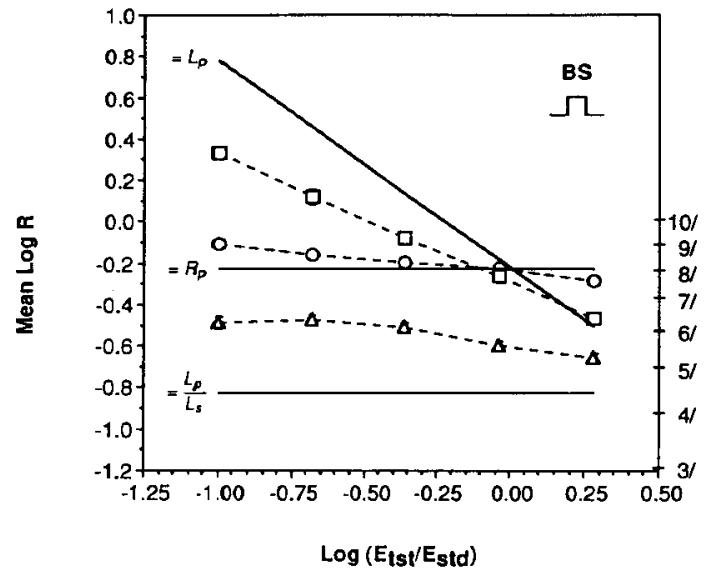

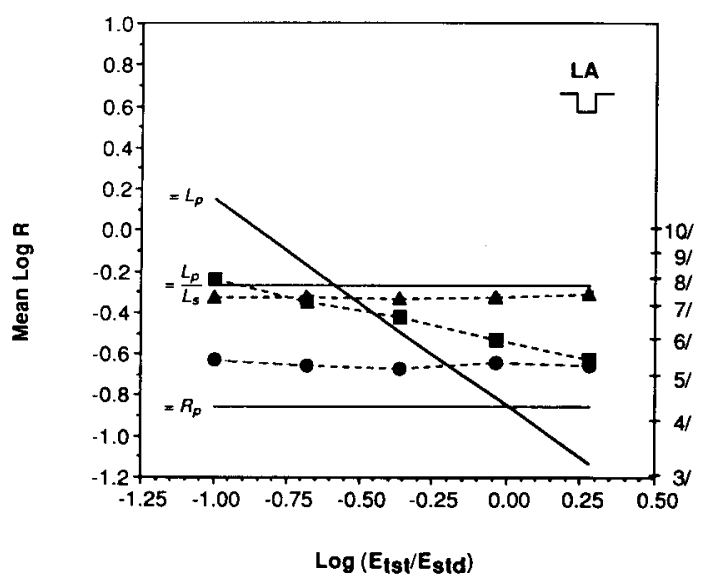

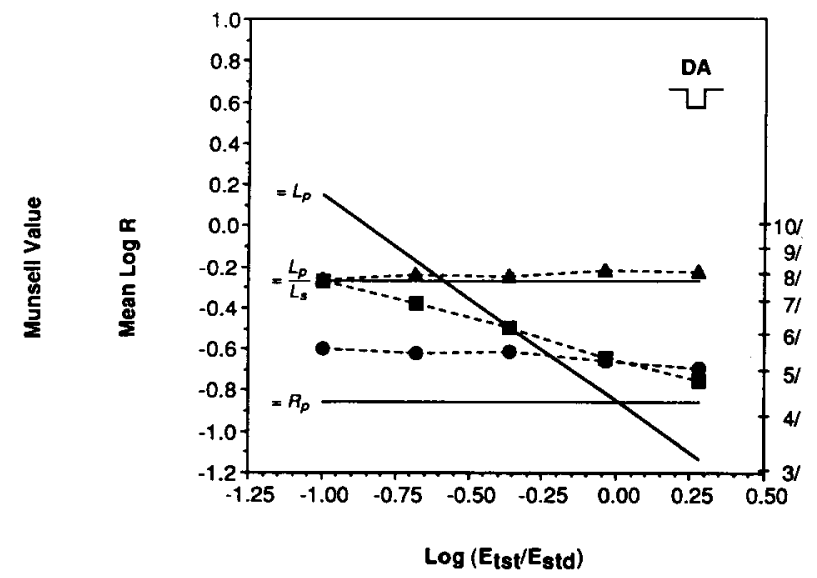

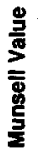

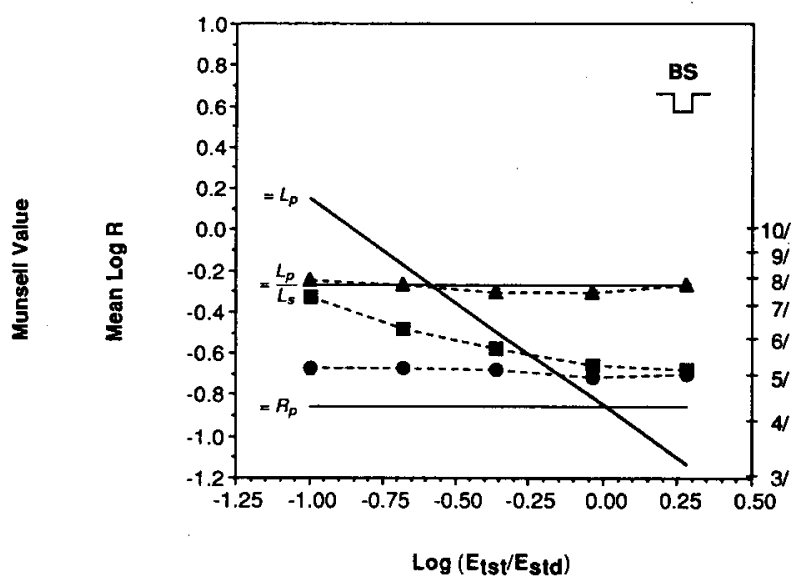

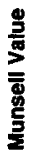

Figure 3. Mean log reflectance data for unequal surround reflectances, Observers L.A., D.A., and B.S. Left panels: increments. Right panels: decrements. Solid lines: theoretical lines, explained in text. Circles: lightness matches. Squares: brightness matches. Triangles: brightness-contrast matches. Error bars are $\pm 1 S E(n=5)$. If no bars are visible, $\pm 1 S E$ is smaller than the plot symbol. 
contrast results (e.g., those of Heinemann, 1955). First, consider the increment condition. If the subject sets the reflectance of the test patch to equal that of the standard patch, the patch/surround luminance ratio $\left(L_{\mathrm{p}} / L_{\mathrm{s}}\right)$ is higher in the test array than in the standard array, because of the lower reflectance of the test surround. Surrounds lower in luminance than patches have previously been found to have only small simultaneous contrast influences on patch brightness. Our lightness matches were affected little by the mismatch between the test and standard patch/surround luminance ratios. The results closely resemble those from the control condition increments (Figure 2) and Arend and Goldstein's (1987b) earlier experiments in which the surrounding luminances were equal.

In the decrement condition, on the other hand, the mismatch between the $L_{s} / L_{\mathrm{p}}$ is more important (Figure 3). When the patch reflectances are approximately equal, the test $L_{\mathrm{s}} / L_{\mathrm{p}}$ is much higher than the standard $L_{\mathrm{s}} / L_{\mathrm{p}}$. As in earlier work, higher luminance surrounds depress the response to a lower luminance patch. This should occur in both the test and the standard, but the effect is stronger in the test array with its higher luminance ratio. Consequently, the test patch's reflectance was greater than required with equal-reflectance test and standard surroundsin this case, by about $0.2 \log$ units.

The departure from lightness constancy due to local simultaneous contrast was of approximately constant size over our 19:1 range of illuminances. This has practical implications: it suggests that the effect of an object's moving from one surround to another does not prevent illumination-invariant lightness perception.

\section{Brightness Matches}

The brightness-matching data are represented as squares. The slanted solid lines are theoretical, the loci where test $L_{\mathrm{p}}$ equal the standard $L_{\mathrm{p}}$. The observers' brightness matches were not photometric matches, however. In the control condition, the brightness matches were very similar to those from Arend and Goldstein's (1987b) experiments with equal surround reflectances, lying between the equal-luminance and equal-reflectance theoretical lines, with the matches for increments lying closer to luminance matches than those for decrements.

As with the lightness matches (and for the same reasons), the brightness matches in the increment condition (Figure 3, left panels) were also very similar to those in Figure 2 and to Arend and Goldstein's data.

In the decrement condition (Figure 3, right panels), on the other hand, the difference between the standard and test surround reflectances did have an important effect. A line through the data has approximately the same slope as in Figure 2 but is shifted upward relative to the equalluminance and equal-reflectance theoretical lines. The size of the shift, approximately $0.2 \mathrm{log}$ units, can be seen clearly at the 0.0 abscissa, where the theoretical lines (and the Figure 2 data) intersect.

The slope is also shallower than that for increments, so that the brightness matches are farther from luminance matches. A similar difference of slope was found in Arend and Goldstein's (1987b) experiments.

\section{Brightness-Contrast Matches}

From other investigators' prior theory and data (e.g., Whittle \& Challands, 1969), we anticipated that the local brightness contrast of the test patch/surround would match that of the standard when its patch/surround luminance ratio was approximately equal to that of the standard. Little effect of the surround difference was expected in either polarity of luminance contrast, because the luminance ratios in the test and standard arrays should be nearly equal at the match point for all illuminance conditions.

The data from the brightness-contrast matches are plotted as triangles in Figures 2 and 3. The bottom horizontal solid line in the increment condition (Figure 3, left panels) and the top horizontal solid line in the decrement condition (Figure 3, right panels) are theoretical lines, the loci for which the patch/surround luminance ratio in the test array matched that in the standard array. In the control condition (Figure 2), the patch/surround luminance ratios were equal when the patch reflectances were equal, so the equal-luminance ratio and equal-reflectance theoretical lines coincide.

Our ratio-match expectation was approximately confirmed when the illuminations were equal $\left(\log E_{\mathrm{tst}} / E_{\text {std }}\right.$ $=0.0$ ), but the data systematically departed from the theoretical line for other illuminations. The negative slope in the increments and very slight positive slope in the decrements occurred consistently for all 3 subjects. A possible explanation is offered below, in the discussion of Brightness Contrast and Weber's Law.

\section{DISCUSSION}

\section{Lightness Constancy and \\ Local Luminance Contrast}

The preceding experiment shows definitively that a strict version of Wallach's edge ratio hypothesis is simply wrong. Local luminance contrast across an edge does not specify the reflectance of the areas abutting the edge (Figure 3), and perceptually it does not determine their lightnesses. Instead, local brightness contrast, a relational perceptual quantity, is more closely associated with local luminance contrast, a relational physical quantity.

The visual system described by our data deals with environmental luminance gradients in a much more sophisticated manner than is supposed in Wallach's hypothesis. Our observers' lightness matches were illumination invariant even when the local luminance contrasts at the target edge were unequal. Furthermore, the systematic departures of the lightness and brightness matches from the theoretical lines due to differences in surround reflectance were independent of illumination.

Even in scenes with a single uniform illumination (very rare in three-dimensional scenes), the relationship between the reflectances of two separated patches cannot be determined from the luminance ratios at the edges of the 
patches alone. It requires that one also know the relationship between the reflectances of the patches' immediate surrounds, which requires assessment of all the luminance gradients lying between the patches. Luminance contrast information from all the luminance gradients lying between the two areas in question must be integrated if their reflectances are to be compared (Arend, in press-b; Arend, Buehler, \& Lockhead, 1971; Arend \& Goldstein, 1987a; Gilchrist, et al., 1983; Land \& McCann, 1971).

In the more common situation of a scene with spatial illumination gradients as well as reflectance gradients, there is another requirement. The luminance gradients must be vector analyzed into their illumination and reflectance components prior to combination of the reflectance gradients. The vector analysis must be based on largescale structure, because the gradient components are often locally indistinguishable (e.g., in the case of cast shadows, or the occlusion of objects in different illuminations). Some preliminary ideas about how this might be accomplished are discussed elsewhere (Adelson \& Pentland, 1991; Arend, in press-b; Barrow \& Tenenbaum, 1978; Gilchrist, et al., 1983; Kersten, 1991).

\section{Generic Viewpoint}

Why can't the Mondrian be "seen as" an ordered set of illuminations on a single uniform reflectance? In fact, a multitude of combinations of local illuminations, reflectances, and shading might produce exactly the same pattern of luminances. Although there is no proof yet, the answer seems to lie in the relative improbability of encountering such a pattern of illuminations on a uniformly colored flat surface in the real world. The improbability comes from both the geometrical arrangement and the variety and range of perceived luminances. It would require a very precise configuration of many light sources and shadow casters to create the Mondrian's luminances. The Mondrian could arise from three-dimensional shading only if the supposed uniform surface were discontinuous in depth ("cut") at some of the edges of the patches, and if the patches were tilted at the angles with respect to the illuminant that were required by their perceived luminances. It is even less likely that conditions external to the surface would produce such a variety of sharp-edged illumination patches on a flat, uniform-reflectance surface.

It has been argued in the literature on both human and machine vision (Ittelson, 1960; Malik, 1987; Nakayama \& Shimojo, 1992) that, given several possible physical causes of the retinal images, the visual system seems to prefer to perceive the one that is the most "generic" and least "special." These generic viewpoint arguments are a particular elaboration of the concept that the visual system perceives the subjectively most probable physical situation (Arend, in press-b; Gregory, 1970; Helmholtz, 1910/1962; Rock, 1983).

According to this point of view, the Mondrian makes our stimuli less ambiguous by making one physical interpretation of the luminance changes more likely than the other possibilities.

\section{Brightness Contrast and Weber's Law}

The brightness difference produced by a constant patch/surround luminance ratio did vary systematically with illuminance. A fixed luminance contrast produced less brightness difference at lower mean luminances.

It is likely that the explanation of this pattern is to be found in the failure of proportional encoding of luminance contrast at these low photopic mean luminances. At high mean luminances, Whittle and Challands (1969) found that Weber's law held for both contrast thresholds and suprathreshold brightness-contrast matches; that is, targets with equal local luminance contrasts produced equal brightness contrasts. ${ }^{2}$ At lower mean luminances, however, constant brightness contrast required higher luminance contrasts. Whittle and Challands's contours of constant brightness contrast closely resembled curves that describe neural adaptation processes in mammalian retinas (Shapley \& EnrothCugell, 1984); they were both well fit by Stiles's template. These and similar data from other laboratories suggest that early visual processes succeed in approximately encoding local luminance contrasts at high mean luminances, but that they result in relative underestimation of luminance contrasts at low mean luminances. As our independent variable, the illuminance of the test array, decreased, the test array moved to the left on Whittle and Challands's abscissa while the standard array stayed fixed. The slight slopes of our data represent a progressive decrease in the brightness contrast produced by a constant luminance contrast as the mean luminance of the test array decreases, in accord with this hypothesis. In experiments reported elsewhere (Arend, 1990b, in press-a), we measured brightness contrast at still lower mean luminances and found further departures from the equal-luminance-ratio line, as expected on the basis of this hypothesis.

One might argue that decreased efficiency of luminance contrast at low mean luminances can also affect our other matching criteria. It is not clear whether this is the case for brightness. It does not seem to be true for lightness. Arend and Goldstein (1987b) found that lightness curves for patch/surround stimuli diverged at low mean luminances, as loss of contrast efficiency would predict. However, we argue (with confirming data) in the next paper of this series (Arend \& Spehar, 1993) that subjects following lightness-matching instructions with simple patch/ surround stimuli like Arend and Goldstein's are able only to match brightness contrasts. With patterns sufficiently complicated to support clear perception of a gray scale (the Mondrian condition), Arend and Goldstein found no convergence of the lightness-matching curves. This would seem to conflict with the hypothesis of low luminancecontrast efficiency at low mean luminances, but there is a relatively simple explanation. When the test and standard patches are embedded in Mondrians, their apparent gray values (lightnesses) no longer depend solely on their luminance contrasts with the immediately surrounding patches. Instead, the Mondrian is perceived as defining a gray scale, and the lightness of the test patch is evaluated in relation to that gray scale. When the test illumi- 
nation is reduced, it affects the entire test Mondrian. The brightness range spanned by the entire test Mondrian is easily perceived to be smaller than that in the standard Mondrian, even though it still appears to range from black surfaces to white surfaces. As a result, it is possible for the test patch to maintain its relative position within the gray scale of the test Mondrian, even though the difference between its brightness and that of the immediately surrounding patches is reduced. This result, too, was confirmed at still lower illuminations (Arend, 1990b, in press-a). Lightness constancy was good even as local brightness contrasts were departing dramatically from the equal-luminance-ratio line. Gilchrist and Jacobsen (1983) studied displays in which physical contrast was reduced by a veiling luminance and found a similar combination of good lightness constancy and reduced apparent contrast.

These conclusions hold over our 19:1 range of illuminances and 33:1 range of reflectances, spanning a range from low photopic to high mesopic mean luminances, the luminances most frequently encountered on visual displays and in most scenes.

\section{Relation to Classical Adaptation Experiments}

Several aspects of our new paradigm make it difficult to compare our data to conventional adaptation data and theory. The test and standard patterns were continuously presented to both eyes, the observers moved their eyes voluntarily back and forth between the test and standard patches, and the test and standard patterns each consisted of a complicated geometry of 29 luminances. Each of these represented some loss of control of the spatiotemporal pattern of light on the observer's retina.

The listed features were careful choices dictated by differences between our goals and those motivating most conventional adaptation research. In the laboratory, use of simple patterns, controlled timing of stimuli, and voluntary fixation allows fairly precise control of retinal stimulation. This is essential for experiments intended to describe low-level sensory processes that might be correlated with identifiable neurophysiological mechanisms and anatomical properties. On the other hand, natural perception of surfaces and objects occurs under very different conditions. The color of a surface is perceived while it is embedded in an extremely complicated spatial array of other surfaces and regions of illumination. Observers move their eyes over this array in a manner so complicated that it can be predicted only probabilistically. As a consequence, it is seldom possible to construct a defensible explanation of phenomena in natural perception in terms of lowlevel visual mechanisms.

In an attempt to bridge this gap, a number of researchers have done experiments with displays of complexity intermediate between the sensory laboratory and natural scenes. The benefit is that it is easier to see and believe connections between the results and natural perception. The cost is that dozens of experiments are required for one to un- derstand the roles of the many parameters of the more complicated spatiotemporal pattern of retinal stimulation.

It is not possible to give this complicated issue thorough treatment here. It is the main topic of Arend (in press-b). Nevertheless, a few issues can be mentioned briefly as examples.

Our instructions regarding eye movements were an attempt to approximate the sequence of retinal stimulation of an observer comparing two surfaces in different regions of a natural scene. Our subjects tried to move their eyes as instructed, and we believe they did a fairly good job. The low variability of the data is reassuring; it suggests that consistency was achieved with respect to variables important to the observers' functional state of adaptation. Nevertheless, there were certainly trials in which the timing only roughly approximated the prescribed 2 -sec fixations. Suppose, for the sake of argument, that an observer produced 2-sec fixations. Achromatic adaptation processes have time constants ranging from the order of tens of milliseconds to minutes (for a recent review, see, e.g., Hayhoe \& Wenderoth, 1991). Therefore, some processes would have run nearly to completion by the end of each 2 -sec fixation, whereas others would have just begun. This tells us very little. Is the observer's perception of the brightness (lightness, brightness contrast) based on the first $100 \mathrm{msec}$ of the fixation? The last $100 \mathrm{msec}$ ? A weighted average over the $2 \mathrm{sec}$ ? With what temporal weights? Is the alternating sequence of adaptations significant? Reliable answers to these questions can only come from extended series of experiments under controlled timing and fixation and from experiments in which statistics of measured natural eye movements are correlated with performance on brightness-matching and other tasks. The latter type of experiment is technically very demanding and little has been done (see Arend \& Skavenski, 1979, and Steinman, Levinson, Collewijn, and van der Steen, 1985, for examples of correlation of eye and head movements with psychophysical performance).

Some clues to the possible outcomes of such experiments can be obtained from the pilot observations that led us to adopt our procedures. Two second fixations were about the shortest that would allow a confident impression of the patch's brightness. The appearance of dimly illuminated test arrays changed over the course of fixations longer than a few seconds, with brightness and brightness contrast greater at the end. This effect was strong enough to dictate the maximum illumination difference between the test and standard patterns. When the illumination ratio was greater than about 20:1, the observer could not stick to the fixation regimen. For higher ratios, the dimly illuminated test array had very low brightness contrast at the beginning of the fixation, and no clear impression of the test patch's properties could be formed. The rapid increase of apparent contrast toward the end of the $2 \mathrm{sec}$ produced an irresistible desire to prolong fixation of the test array to "get a better look at the test patch." This 
suggests that important adaptive changes occur on the time scale of a few seconds, a range that has received relatively little experimental attention.

Other display parameters present similar difficulties. What spatial properties of the surround are important? What statistics of the Mondrian are important? The average luminance? Averaged with what spatial weighting function? Should we take into account the spatial properties of the retinal region underlying the pattern? Is the relation between the spatial scale of the test patch and that of the Mondrian patches relevant?

We have a little information about the structure of the Mondrian. Lightness matches were little affected by two kinds of changes of the Mondrians (Arend, unpublished). Random rearrangement of the grays in the test Mondrian produced very similar lightness matches. The precise population of grays in the Mondrians is also not critical. Test and standard Mondrians composed of only black and white patches gave very similar lightness matches.

\section{Dependence on Surround Reflectance}

The lightness and brightness matches for increments were only slightly affected by the difference in reflectance of the surrounds of the test and standard patches, but for decrements, the effects were larger. The size of the effect is most clearly seen by comparing Figures 2 and 3 at the 0.0 abscissa, where the test and standard illuminations are equal. For decrements, the subject required that the reflectance of the test patch be higher than the standard reflectance to obtain equal lightness and equal brightness. The surround reflectance is higher for the test patch than for the standard, so this is simply a measurement of the classical simultaneous contrast demonstration. Its effect is a small fraction of the gray scale, about 1.5 Munsell value steps out of the full scale of 10 steps.

Although this error is unlikely to have gross behavioral consequences, it is a deviation from lightness constancy; if the reflectance of the test patch is the same as that of the standard, the lightness and brightness of the test patch are less than those of the standard. The magnitude of this constancy error due to local simultaneous contrast was independent of the illumination of the test. Thus the lightness matches were illumination invariant, but for decrements, they were not lightness constant.

Asymmetries between increments and decrements have also been found in adaptation experiments with simpler stimuli (Whittle, 1992; Whittle \& Challands, 1969; and see Whittle, in press-a, for a thorough review). There is no satisfactory explanation for this at present. There is good reason, however, to think that such asymmetries can be of little importance in natural perception. Very few regions in natural scenes have higher luminance than all of the adjacent regions, and still fewer are completely surrounded by a single luminance. It is biologically adaptive for the influence of the surround on surface color perception to be small. The luminance relations between a surface and its surround(s) is often an accidental property of the scene, of no significance for human behavior.

\section{REFERENCES}

Adelson, E. H., \&entland, A. P. (1991). The perception of shading and reflectance. In B. Blum (Eds.), Channels in the visual nervous system: Neurophysiology, psychophysics and models (pp. 195-208). London: Freund.

AREND, L. E. (1990a). Apparent surface color is more than color appeararce. In M. H. Brill (Eds.), Perceiving, measuring, and using color: Proceedings of the 1990 SPIE/SPSE Symposium on Electronic Imaging (pp. 2-8). Bellingham, WA: SPIE.

AREND, L. E. (19906). Mesopic lightness, brightness, and brightness contrast. In OSA Annual Meeting Technical Digest 1990 (p. 206). Washington, DC: Optical Society of America. (Abstract)

AREND, L. E. (1991). Apparent contrast and surface color in complex scenes. In B. E. Rogowitz, M. H. Brill, \& J. P. Allebach (Eds.), Human vision, visual processing, and digital display: II. Proceedings of the SPIE 1453 (pp. 412-421). San Jose, CA: SPIE

AREND, L. E. (in press-a). Mesopic brightness, lightness, and brightness contrast. Perception \& Psychophysics.

Arend, L. E. (in press-b). Surface colors, illumination, and surface geometry: Intrinsic-image models of human color perception. In $\mathbf{A}$. $\mathbf{L}$. Gilchrist (Eds.), Lightness, brightness, and transparency. Hillsdale, NJ: Erlbaum.

Arend, L. E., Buehler, J. N., LockheAd, G. R. (1971). Difference information in brightness perception. Perception \& Psychophysics, 9, 367-370.

AREND, L. E., GoldsteIN, R. (1987a). Lightness models, gradient illusions, and curl. Perception \& Psychophysics, 42, 65-80.

AREND, L. E., GolDSTEIN, R. (1987b). Simultaneous constancy, lightness and brightness. Journal of the Optical Sociery of America A, 4, 2281-2285.

AREND, L. E., \& GoldsteIN, R. (1990). Lightness and brightness over spatial illumination gradients. Journal of the Optical Society of America $A$, 7, 1929-1936.

Arend, L. E., Reeves, A. (1986). Simultaneous color constancy. Journal of the Optical Society of America A, 3, 1743-1751.

AREND, L. E., SkA veNSKI, A. (1979). Free scanning of gratings produces patterned retinal exposure. Vision Research, 19, 1413-1419.

AREND, L. E., SPEHAR, B. (1993). Lightness, brightness, and brightness contrast: 2. Reflectance variation. Perception \& Psychophysics, 54, 457-468.

Barrow, H. G., \& Tenenbaum, J. (1978). Recovering intrinsic scene characteristics from images. In A. R. Hanson \& E. M. Riseman (Eds.), Computer vision systems (pp. 3-26). Orlando, FL: Academic Press.

Evans, R. M. (1974). The perception of color. New York: Wiley. Gilchrist, A. [L.], Delman, S., \& JaCobSen, A. (1983). The classification and integration of edges as critical to the perception of reflectance and illumination. Perception \& Psychophysics, 33, 425-436.

GilChRIST, A. L., \& JACOBSEN, A. (1983). Lightness constancy through a veiling luminance. Joumal of Experimental Psychology: Human Perception \& Performance, 9, 936-944.

GilChRIST, A. L., \& JACOBSEN, A. (1989). Qualitative relationships are decisive. Perception \& Psychophysics, 45, 92-94.

Gregory, R. L. (1970). The intelligent eye. New York: McGraw-Hill.

HaYhoe, M. \& Wenderoth, P. (1991). Adaptation mechanisms in color and brightness. In A. Valberg \& B. Lee (Eds.), From pigments to perception (pp. 353-367). New York: Plenum.

HeinemanN, E. G. (1955). Simultaneous brightness induction as a function of inducing- and test-field luminances. Journal of Experimental Psychology, 50, 89-96.

Heinemann, E. G. (1989). Brightness contrast, brightness constancy, and the ratio principle. Perception \& Psychophysics, 45, 89-91.

Helmholtz, H. vON (1962). Handbook of physiological optics (3rd ed., J. P. C. Southall, Trans.). New York: Dover. (Original work published 1910) 
Hess, C. , Pretori, H. (1970). Quantitative investigation of the lawfulness of simultaneous brightness contrast (H. R. Flock and J. H. Tenney, Trans.). Perceptual \& Motor Skills, 31, 947-969. (Original work published 1894)

ITTELSON, W. H. (1960). Visual space perception. New York: SpringerVerlag.

JACOBSEN, A., \& Gilchrist, A. [L.] (1988). The ratio principle holds over a million-to-one range of illumination. Perception \& Psychophysics, 43, 1-6.

JAMESON, D., \& HURVICH, L. M. (1989). Essay concerning color constancy. Annual Review of Psychology, 40, 1-22.

KERSTEN, D. (1991). Transparency and the cooperative computation of scene attributes. In M. S. Landy \& J. A. Movshon (Eds.), Computational models of visual processing (pp. 187-208). Cambridge, MA: MIT Press.

KoffKa, K. (1935). Principles of Gestalt psychology. New York: Harcourt, Brace, \& World.

LAND, E. H., \& MCCANN, J. J. (1971). Lightness and retinex theory. Journal of the Optical Society of America, 61, 1-11.

MALIK, J. (1987). Interpreting line drawings of curved objects. International Journal of Computer Vision, 1, 73-103.

MARR, D. (1978). Representing visual information. In A. R. Hanson \& E. M. Riseman (Eds.), Computer vision systems (pp. 61-80). Orlando, FL: Academic Press.

Nakayama, K., \& Shimojo, S. (1992). Experiencing and perceiving visual surfaces. Science, 257, 1357-1363.

Rock, I. (1983). The logic of perception. Cambridge, MA: MIT Press.

Shapley, R., \& ENroth-Cugell, C. (1984). Visual adaptation and retinal gain controls. Progress in Retinal Research, 3, 263-346.

Steinman, R. M., Levinson, J. Z., Collewisn, H., \& van der STEEN, J. (1985). Vision in the presence of known natural retinal image motion. Journal of the Optical Society of America A, 2, 226-233.

TAKASAKI, H. (1966). Lightness change of grays induced by change in reflectance of gray background. Journal of the Optical Society of America, 56, 504

Wallach, H. (1948). Brightness constancy and the nature of achromatic colors. Journal of Experimental Psychology, 38, 310-324.

WALLACH, H. (1976). On perception. New York: Quadrangle/The New York Times Book Co.

WhitTle, P. (1992). Brightness, discriminability and the "Crispening Effect." Vision Research, 32, 1493-1507.

WhitTle, P. (in press-a). Contrast-brightness and ordinary seeing. In
A. L. Gilchrist (Eds.), Lightness, brightness, and transparency. Hillsdale, NJ: Erlbaum.

WhitTle, P. (in press-b). The psychophysics of contrast-brightness. In A. L. Gilchrist (Eds.), Lightness, brightness, and transparency. Hillsdale, NJ: Erlbaum.

Whittle, P., \& Challands, P. D. C. (1969). The effect of background luminance on the brightness of flashes. Vision Research, 9, 1095-1110.

\section{NOTES}

1. In this context, these should not be interpreted as accurately indicating equal lightness steps (or any other psychological property of the Munsell value scale), since our viewing conditions differed from those used to generate the Munsell scale. They are presented as an additional physical specification of our stimuli, the Munsell papers we would have had to use had we chosen to create our stimuli with papers and lights rather than on a CRT.

2. To simplify the explanation, we use brightness contrast here to describe the results of Whittle and Challands's (1969) experiments. This usage involves a minor assumption. They presented small test and comparison fields on circular surround fields to the right and left eyes, respectively. The targets were positioned so that the surround fields were haploscopically fused, with the test and comparison patches appearing side by side, with a small separation. Their subjects were asked to match the brightnesses of the test and comparison patches. The fused surrounds had a unified brightness. Thus, when the brightnesses of the two patches were equal, the brightness difference between each patch and the common surround was the same. However, Whittle and Challands's subjects gave slightly different matches when asked to equate the edge contrasts of the patches. By using the term brightness contrast, we are assuming that the physical contrast setting that made the brightness of Whittle and Challands's test patch match that of the comparison patch would produce an approximate brightness-contrast match under normal viewing conditions, in which the surrounds of the two patches were presented side by side rather than fused. No experiments have yet been done to check this assumption directly. Whittle (in press-a) has decided to give a new name, contrast brightness, to the matches that Whittle and Challands called brightness matches. He discusses the relationship among the various tasks at length (Whittle, in press-b).

(Manuscript received March 11, 1992; revision accepted for publication February 26, 1993.) 